\title{
The JUNO double calorimetry system
}

\author{
Jilei Xu*, on behalf of the JUNO collaboration \\ Institute of High Energy Physics, Chinese Academy of Sciences \\ E-mail: xujl@ihep.ac.cn
}

\begin{abstract}
The Jiangmen Underground Neutrino Observatory (JUNO) detector was designed to achieve 3\% energy resolution which required 18,000 high quantum efficiency 20-inch PMTs (LPMTs) closely packed around the liquid scintillator (LS) target ball. Besides this, 25,000 3-inch PMTs (SPMTs) were also designed to be installed between the LPMTs gaps forming a double calorimetry system to improve and extend JUNO physics reach, with a better energy resolution, muon reconstruction, supernova neutrino detection and so on. The LPMTs have been produced by the Chinese NNVT (15,000 PMTs) and by the Japanese Hamamatsu Photonics company; the 3" PMTs are made by the HZC company. Until recently more than 15,000 LPMTs and SPMTs have been produced, respectively. Performance test data so far indicate that both LPMTs and SPMTs perform as expected. Both system's high voltage divider, potting, cabling, connector are in the production phase. The electronics and underwater box are all making good progress.
\end{abstract}

The 21st international workshop on neutrinos from accelerators (NuFact2019) August 26 - August 31, 2019

Daegu, Korea

${ }^{*}$ Speaker. 


\section{Introduction}

JUNO [1] is a neutrino oscillation experiment with a $53 \mathrm{~km}$ distance from reactors and a 700m overburden, currently under construction in southern China. The primary goal is to measure the neutrino mass hierarchy with better than $3 \sigma$ after 6 years of data taking [2]. Therefore high transparency liquid scintillator (LS), high (75\%) coverage of photomultiplier tubes and low backgrounds are needed to achieve energy resolution of $3 \% / \sqrt{E}$ and calibration error lower than $1 \%$. This is the most challenging design in the reactor neutrino experiments throughout the world.

The center target ball is 20 kton LS filled in an acrylic shell with inner diameter $35.4 \mathrm{~m}$, which is immersed in a cylinder water pool with diameter and height both $43.5 \mathrm{~m}$ (Figure 1). There are 18,000 high quantum efficiency (QE) 20-inch PMTs (LPMTs) closely packed around LS ball. Beside this, 25,000 3-inch PMTs (SPMTs) were also designed to install between the gaps of LPMTs (Figure 1, right) to make a double calorimetry system to improve and extend JUNO physics, which have been proposed in 2014 [3].

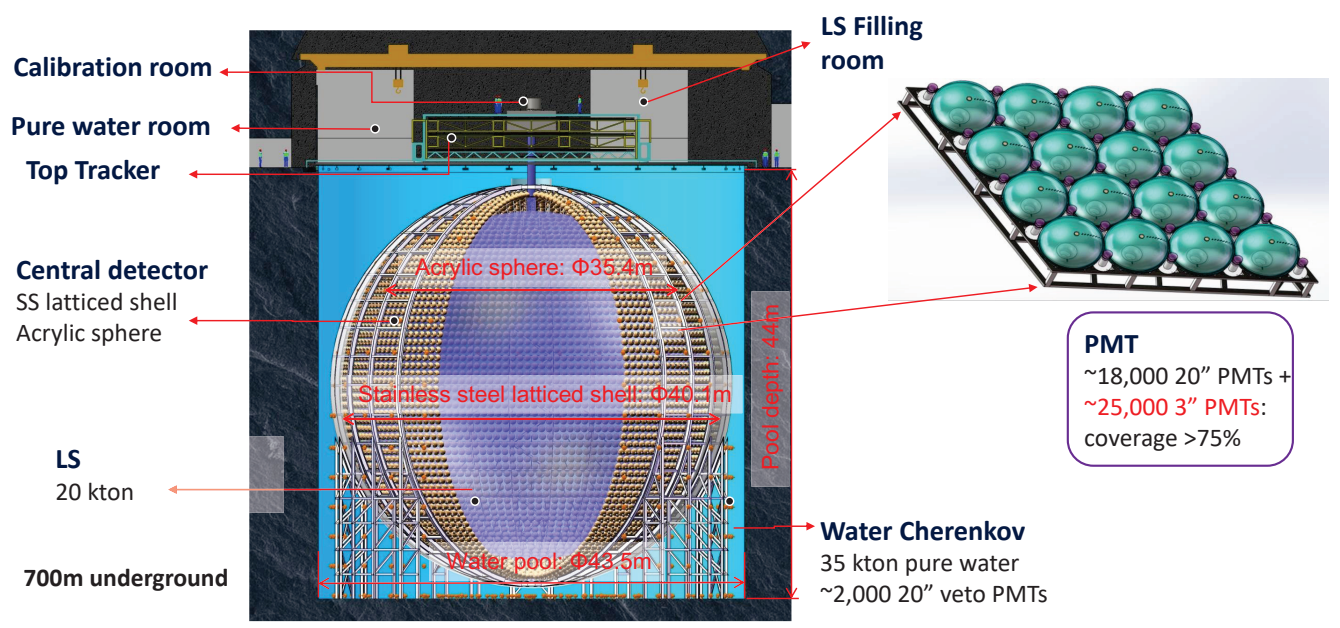

Figure 1: JUNO detector design

\section{Double calorimetry system}

LPMT and SPMT system both will detect the same IBD signals, but SPMT area is about 40 times smaller than LPMT and will be almost always work in single photoelectron (SPE) mode for IBD signals. LPMT measures energy via "charge integration" increase photon statistics mainly contribute to stochastic term ( $a$ term in equation 2.1) and SPMT measures energy via "photon counting" control systematics contribute to non-stochastic term ( $b$ term in equation 2.1 ).

$$
\frac{\sigma(E)}{E}=\sqrt{\left[\frac{a}{\sqrt{E}}\right]^{2}+[b(E)]^{2}}
$$

As a result, it will help constrain the systematics in the LPMT energy reconstruction, improving the energy resolution and the sensitivity of neutrino mass hierarchy measurement. They will also improve the muon reconstruction resolution, help reduce muon-related isotope backgrounds, 
provide an independent measurement of the $\theta_{12}$ and $\Delta m_{21}^{2}$ solar parameters with unprecedented precision, and improve the measurement of supernova neutrinos.

For LPMTs, 15,000 Micro Channel plate PMTs are produced by North Night Vision Technology Co. (NNVT) and 5,000 dynode PMTs are producing by Japanese Hamamatsu Photonics $[4,5]$. Concerning the SPMTs, all 25,000 PMTs (XP72B22) are produced by Hainan Zhanchuang Photonics Co. (HZC) [6]. Until now more than 15,000 LPMTs and SPMTs have been produced respectively. Performance test data indicates that both LPMTs and SPMTs perform as expected.

\section{Small PMT system}

Figure 2 shows one group of under water box (UWB) with 8 connectors, $16 \times 8=128$ PMTs and cables. In one UWB, there are two high voltage (HV) splitter boards to split HV and signal, one multichannel front-end electronics to read out the charge and time of PMT signal, one control unit, $16 \mathrm{HV}$ units (50\% redundancy) and one bellows with power, data cables connecting to the surface data acquisition (DAQ). JUNO will install 200 UWBs.

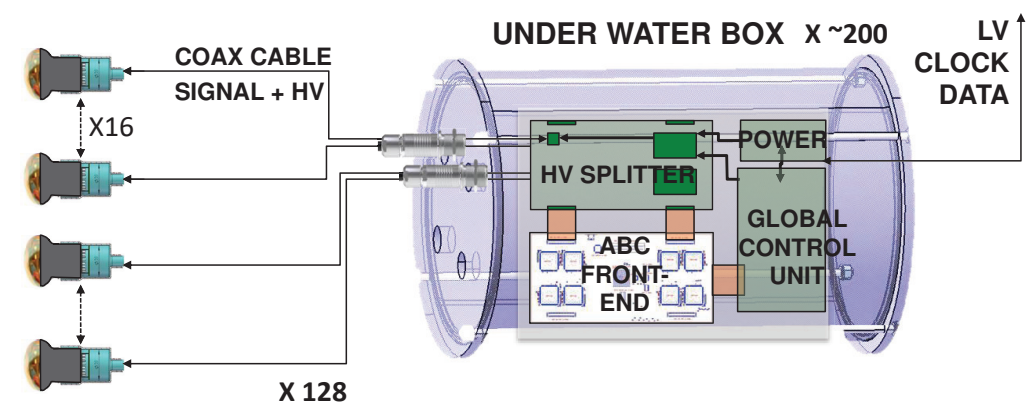

Figure 2: Small PMT system

The mass production of SPMT began in January, 2018, with a production speed of about 1,000 pcs / month. Currently, 19,000 out of 26,000 SPMTs were already produced. JUNO have tested 15,000 PMTs, and only a few of PMTs were rejected and almost all the PMTs ( $>99 \%)$ can satisfy JUNO requirements (Figure 3). Not only the value should be in the range limitation, but also $f$ or each batch of acceptance the average value also should satisfy our requirement (Table1).

The PMT high voltage divider (Figure 4) is under mass production, more than 6,000 pieces have been produced. Potting technology has been developed successfully and delivered to the company which will do the mass potting soon. The water proofing cable and connector was developed by AXON France and China which is ready to start the production soon.

About 3,000 CatiROCs [7] were produced for electronics. The characterization, reliability, long-term stress, control and mockup test show good performances of the splitter and HV modules. The UWBs are designed to withstand pressures up to $50 \mathrm{~m}$ depth for 20 years.

\section{Summary}

The SPMTs as an independent PMT system and with the LPMTs makes double calorimetry system, can detect the same IBD signals and improve the energy reconstruction in synergy with 

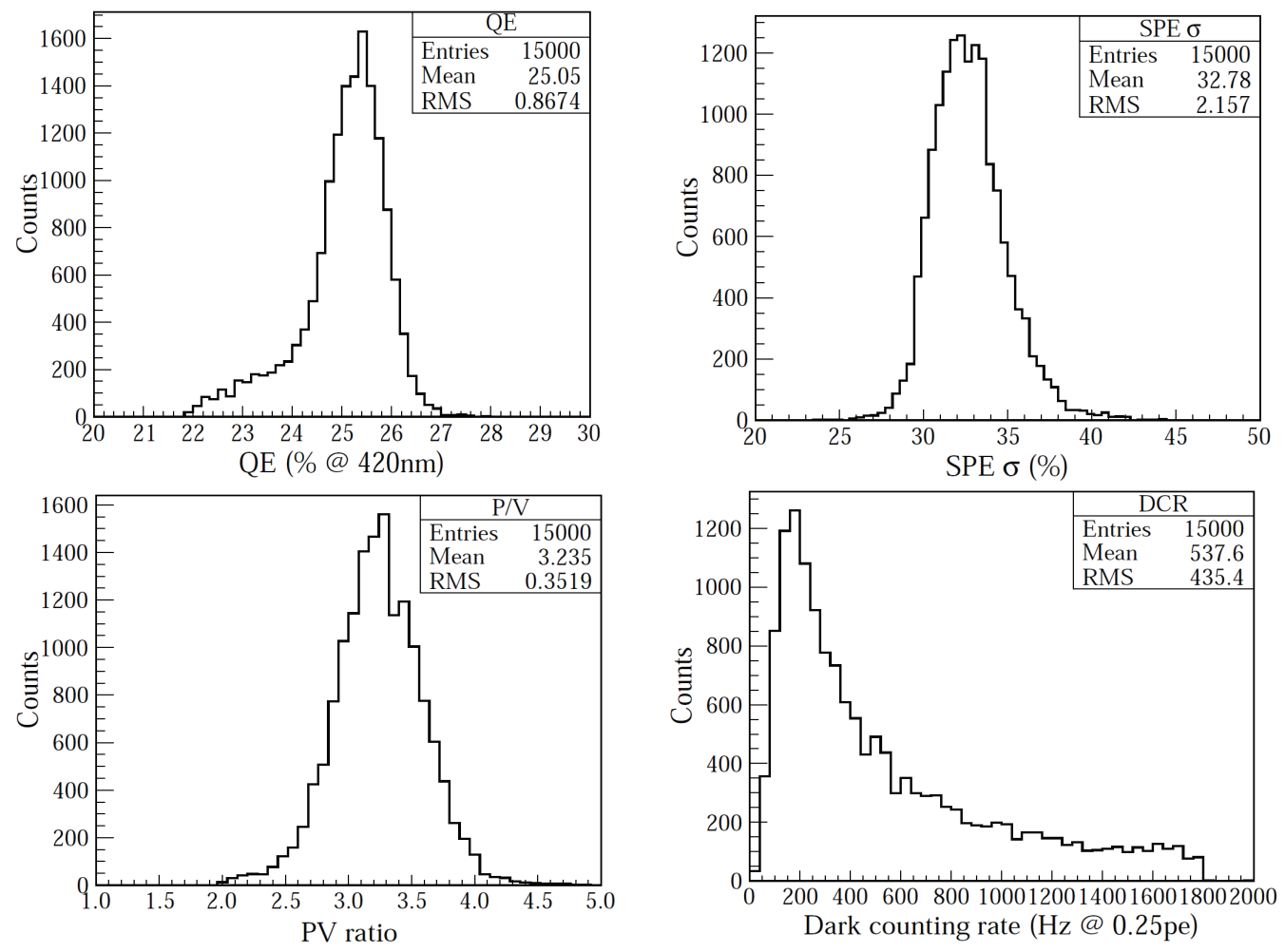

Figure 3: 15,000 accepted PMT performance: QE, single photon electron resolution (SPE $\sigma$ ), peak valley (PV) ratio and dark counting rate.

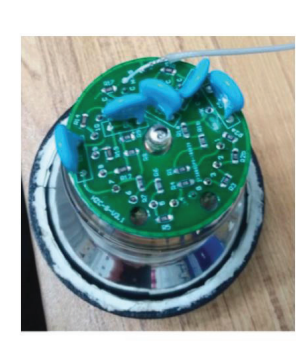

(a)

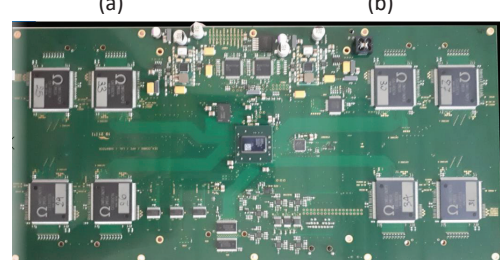

(d)

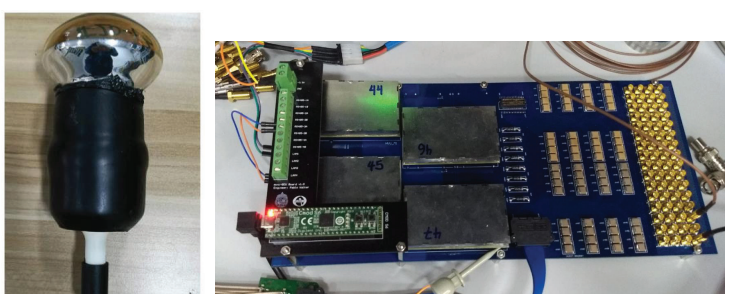

(C)

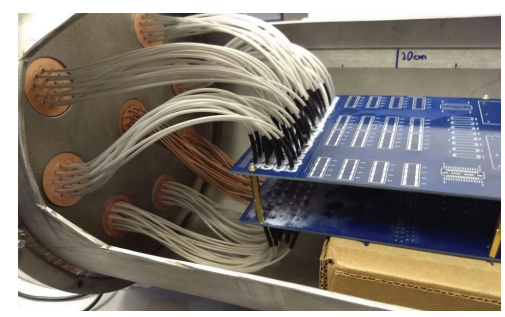

(e)

Figure 4: The real pictures of each part. (a) Divider; (b) Potting; (c) HV splitter board; (d) Electronics (V1); (e) Mock up test of UWB.

the LPMTs, aiming to reach $3 \% / \sqrt{E}$ and $1 \%$ energy scale and enhanced physics capabilities. Until now more than 15,000 LPMTs and SPMTs have been produced. Performance test data indicate that both LPMTs and SPMTs perform as expected. Both system's high voltage divider, potting, 
Table 1: Summary of bare PMT acceptance test.

\begin{tabular}{ccc}
\hline Parameters & Sampling requirements & Result (Mean) \\
\hline$\Phi($ Glass bulb) & {$[78,82] \mathrm{mm}$} & OK \\
QE @ 420 nm & $>22 \%(>24 \%)$ & $25.1 \%$ \\
HV @ gain $3 \times 10^{6}$ & {$[900,1300] \mathrm{V}$} & $1121 \mathrm{~V}$ \\
SPE resolution & $<45 \%($ Mean $<35 \%)$ & $32.8 \%$ \\
PV ratio & $>2($ Mean $>3)$ & 3.2 \\
DCR @ 0.25 PE & $<1.8 \mathrm{kHz}(\mathrm{Mean}<1 \mathrm{kHz})$ & $538 \mathrm{~Hz}$ \\
DCR @ 3.0 PE & $<30 \mathrm{~Hz}$ & $7.2 \mathrm{~Hz}$ \\
TTS (FWHM) & $<5 \mathrm{~ns}$ & $4.9 \mathrm{~ns}$ \\
Pre-pulse (10 ns - 90 ns) & $<5 \%($ Mean $<4.5 \%)$ & $0.4 \%$ \\
After-pulse (50 ns - 20 $\mu \mathrm{s})$ & $<15 \%($ Mean $<10 \%)$ & $4.8 \%$ \\
QE non-uniformity & $<11 \%$ & $5.2 \%$ \\
$\Phi$ (eff. cathode) & $>74 \mathrm{~mm}($ Mean $>76 \mathrm{~mm})$ & $77.1 \mathrm{~mm}$ \\
QE @ 320 nm & $>5 \%$ & $13.4 \%$ \\
QE @ 550 nm & $>5 \%$ & $8.8 \%$ \\
\hline
\end{tabular}

cabling are ready for production. The splitter, electronics and underwater box are in good progress.

\section{Acknowledgments}

This work was supported by the National Natural Science Foundation of China No. 11975258 and 11605222, the Strategic Priority Research Program of the Chinese Academy of Sciences, Grant No. XDA10011200, and Special Fund of Science and Technology Innovation Strategy of Guangdong Province.

\section{References}

[1] T. Adam et al., arXiv:1508.07166v2 [physics.ins-det] (2015).

[2] F. An et al., J. Phys. G: Nucl. Part. Phys. 43, 030401 (2016).

[3] Miao He, Radiation Detection Technology and Methods, 2017, 1 (2) : 21.

[4] Zhonghua Qin, Proceedings of International Conference on Technology and Instrumentation in Particle Physics 2017, Springer Proceedings in Physics, 213 : 285 - 293. Springer, Singapore.

[5] Anfimov N., Journal of Instrumentation, 2017, 12 (06) : C06017.

[6] Nan Li et al., Radiation Detection Technology and Methods (2019) 3 : 6.

[7] S. Conforti et al., Proceedings of International Conference on Technology and Instrumentation in Particle Physics 2017, Springer Proceedings in Physics, 212 : 168 - 172, Springer, Singapore. 